\title{
Linx
}

Revue des linguistes de l'université Paris X Nanterre

$45 \mid 2001$

Invariants et variables dans les langues. Études typologiques

\section{Typologie des oppositions aspectuelles}

Jack Feuillet

\section{OpenEdition}

Journals

Édition électronique

URL : http://journals.openedition.org/linx/832

DOI : 10.4000/linx.832

ISSN : 2118-9692

Éditeur

Presses universitaires de Paris Nanterre

\section{Édition imprimée}

Date de publication : 1 décembre 2001

Pagination : 139-146

ISSN : 0246-8743

\section{Référence électronique}

Jack Feuillet, «Typologie des oppositions aspectuelles », Linx [En ligne], 45 | 2001, mis en ligne le 22 juin 2012, consulté le 10 décembre 2020. URL : http://journals.openedition.org/linx/832 ; DOI : https:// doi.org/10.4000/linx.832

Ce document a été généré automatiquement le 10 décembre 2020.

Département de Sciences du langage, Université Paris Ouest 


\title{
Typologie des oppositions aspectuelles
}

\author{
Jack Feuillet
}

1 L'aspect est l'une des trois grandes catégories verbales, d'un point de vue sémantique, avec le temps et le mode. Il représente la vision la plus objective dans la mesure où le procès est considéré en soi ou selon divers découpages ou phases, comme le déroulement ou l'achèvement. Il est donc indépendant de sa situation dans le temps ou de sa réalité. Il porte avant tout sur le noyau de l'unité verbale, c'est-à-dire en termes traditionnels le verbe. Cependant, son impact, dans certains cas, s'étend à l'objet ou aux objets.

2 L'exposé sera divisé en trois parties. La première concerne la problématique générale de l'aspect, en particulier sa distinction d'autres concepts proches, mais qui, d'un point de vue méthodologique, doivent être séparés. Ensuite, il conviendra de considérer les diverses oppositions aspectuelles en évitant les assimilations qu'une analyse rapide ne manquerait pas de faire. Enfin, on dira un mot des problèmes connexes de l'aspect, d'une part les valeurs dites "complémentaires", d'autre part les traits externes qui ne s'accordent pas avec les différents découpages du procès.

\section{Méthodologie de l'aspect}

\subsection{Aspect et aktionsart}

3 Ce sont les linguistes allemands qui, les premiers, ont introduit la distinction entre Aspekt et Aktionsart. Selon Ulrich (1972: 16 et 12), l'aspect est « die Verlaufsweise eines verbalen Geschehens [...] im Hinblick auf sein Verhältnis zum Zeitverlauf» et l'Aktionsart « die Verlaufsweise, Spielart eines verbalen Geschehens im Blick auf die Art seiner inhaltlichen Modifizierung ». Autrement dit, l'aspect est la manière de considérer le procès dans son déroulement temporel, tandis que l'Aktionsart considère le procès du point de vue de sa modification (sémantique) interne. Malheureusement, dans les exemples donnés par l'auteur, on constate une confusion préjudiciable des deux concepts, car, outre l'assimilation abusive de duratif et d'imperfectif, ainsi que de ponctuel avec perfectif, les 
illustrations sont fournies par des verbes différents : schlafen "dormir» / einschlafen «s'endormir », essen " manger » / aufessen «manger tout, achever (de manger) ». C'est justement cette confusion qu'il faut absolument éviter: l'aspect est une catégorie grammaticale et s'exprime par différentes formes d'un même verbe. À partir du moment où l'on oppose deux verbes, même s'ils sont formés sur la même racine, on quitte le domaine de la grammaire pour entrer dans celui du lexique. Or, les liens qui existent par exemple entre un verbe simple et un verbe composé (fleurir / défleurir ; écrire / décrire) ne peuvent en aucun cas être traités par l'aspectologie proprement dite. On pourrait remplacer Aktionsart par aspectivité, et englober les deux termes sous la notion générale d' aspectualité (sur le modèle de temporalité), qui peut avoir son utilité dans une perspective sémantique globale.

\subsection{Sémantisme du verbe}

4 La deuxième confusion à éviter est celle qui consiste à définir les oppositions aspectuelles à partir du sémantisme des verbes. On ne compte plus les études qui parlent d'aspect duratif pour des verbes comme dormir, chercher et d'aspect ponctuel pour des verbes comme entrer, trouver. Comme ces mêmes notions sont également utilisées pour expliquer par exemple la différence entre l'imparfait et le passé simple, on aboutit à des formulations contradictoires du genre : il chercha longtemps la solution à son problème serait un duratif ponctuel, tandis que il trouvait toujours le temps pour sortir avec ses amis serait un ponctuel duratif. Et l'on chercherait en vain ce qui dure le plus longtemps dans Une heure sonnait lorsqu'il entra.

Dans cet ordre d'idées, il faut exclure aussi le sémantisme des circonstants pour définir l'aspect. Par exemple, sous l'entrée ASPECT dans le dictionnaire Le Langage (1973 : 38), on trouve la phrase suivante: "Dans le cas de l'aspect discontinu (on parle aussi d'aspect itératif), le procès se répète: Il se lève tous les jours à huit heures ». Or, il est clair que la répétition est exprimée par tous les jours à huit heures, et non par la forme verbale. Par conséquent, il est tout à fait exclu de parler d'aspect dans ce cas-là, puisque ce n'est pas la " grammaire » du verbe qui est en cause. Le même ouvrage précise qu'« on peut parler d'inchoatif et d'aspect terminatif lorsqu'on envisage le début ou la fin du procès; le français utilise des périphrases du type : je commence à, il se met à (infinitif) ou il finit de. Là encore, on peut se demander si l'on a le droit de parler d'aspect à partir du moment où l'on n'accorde pas à commencer ou à finir le statut d'auxiliaires. Il en serait de même avec en train de: s'il est vrai que cette périphrase correspond à l'une des valeurs de la forme progressive en -ing de l'anglais, elle ne jouit pas du même statut. Par conséquent, avant de se livrer à une étude de sémantique générale, il faut d'abord considérer la structure spécifique de chaque système.

\subsection{Aspect et sous-aspect}

6 Cette question de la constitution spécifique de chaque système explique qu'une opposition aspectuelle qui se trouve dans une langue donnée peut ne pas avoir le même statut dans une autre langue. Par exemple, en grec ancien, on trouve trois thèmes principaux : celui du présent, celui de l'aoriste et celui du parfait. Cette opposition ternaire traverse tout le système, puisqu'elle est représentée à l'indicatif, au subjonctif, à l'optatif, à l'infinitif et aux participes. En revanche, le passé simple français qui, par bien des côtés, 
se comporte comme l'aoriste de l'indicatif du grec, est isolé puisqu'il n'est attesté qu'à l'indicatif. Il y a donc intérêt à réserver le terme d'aspect pour une opposition qui traverse tout ou presque tout le système, et à parler de sous-aspect lorsque l'opposition n'est pas générale. C'est ce que l'on fait par exemple en slavistique avec une série de verbes de mouvement (russe idti / xodit' « aller ») qui sont imperfectifs et qui sont opposés à un seul perfectif. Même si ce cas de figure est particulièrement clair, il n'est pas impossible de déterminer à chaque fois ce qui ressortit à l'aspect et ce qui appartient au sous-aspect.

7 Ces précautions méthodologiques sont indispensables en aspectologie: si on ne les observe pas, on court le risque grave de tout mélanger. En effet, il est bien rare qu'à un moment ou à un autre de la description n'interviennent pas des considérations d'ordre lexical qui faussent la perspective.

\section{Types d'oppositions aspectuelles}

8 Si les difficultés méthodologiques sont importantes, elles ne le cèdent en rien à la profusion terminologique. Voici, à titre d'illustration, les termes que l'on rencontre dans les grammaires et autres ouvrages de linguistique. On commencera par les oppositions binaires :

- accompli / inaccompli (ou non-accompli)

- imperfectif / perfectif ; latin infectum / perfectum.

- complétif / incomplétif.

- progressif / non-progressif.

- continu / non-continu ou momentané.

- duratif / ponctuel.

- télique / atélique.

- indéfini / défini.

- indéterminé / déterminé

- résultatif / non-résultatif.

Comme termes n'entrant pas dans une opposition binaire, on trouve : inchoatif, inceptif, ingressif, fréquentatif, habitatif, habituel, itératif, répétitif, continuatif, cursif, complexif, égressif, terminatif, semelfactif. On ajoutera les termes qui se retrouvent dans la désignation de temps grammaticaux, tels que parfait et aoriste.

Il est évident qu'une telle prolifération n'est pas due tant aux données qu'à des types d'analyse différents et qu'on doit retrouver, sinon des phénomènes identiques, du moins des phénomènes semblables. L'homonymie est évidente pour progressif et continu, de même que pour habituel, fréquentatif ou habitatif ou pour itératif et répétitif. La distinction entre inchoatif, inceptif et ingressif ne saute pas aux yeux, et il faut regarder de plus près ce que peut recouvrir la distinction égressif / terminatif. Bref, la typologie de l'aspect aurait d'abord besoin d'une sérieuse remise en question des concepts.

11 Il faut se pencher sur la nature des oppositions aspectuelles et voir les différentes manières dont peut être envisagé un procès. Si on représente ce dernier sous la forme d'un segment $A B$ sur une ligne $x y$ du temps, on peut envisager les possibilités suivantes :

12 1) Soit le procès $A B$ est saisi comme un tout, et l'on aura affaire à un complexif. (schéma $\mathrm{N}^{\circ}$

1). Ce terme peut être employé à la place de complétif, qui suppose la contrepartie négative d'incomplétif. Or, cette dernière notion est vague, et surtout il ne semble pas qu'il 
existe de systèmes verbaux bâtis de cette manière. De cette façon, on peut réserver le terme aoriste à la désignation d'un temps grammatical, bien que les grammaires descriptives du grec ancien parlent d'infinitif ou de participe aoriste. Les descripteurs du grec ancien insistent par exemple sur le fait qu'en dehors de l'indicatif, les formes aorististiques expriment l'idée pure, sans considération de durée. Bien que la différence entre les formes de présent et celles d'aoriste ne soit pas toujours facile à saisir, il semble que le présent soit le terme non marqué, l'aoriste exprimant, comme le dit Humbert (1972 : 172), « l'entrée brusque dans un état nouveau » (au participe).

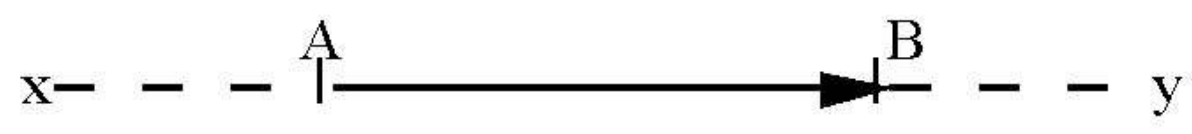

2) Soit le procès $A B$ est achevé, et l'on a ce cas une opposition binaire accompli / nonaccompli, le point $\mathrm{B}$ servant de limite entre les deux (schéma $\mathrm{n}^{\circ} 2$ ). Elle peut être illustrée par le français chanter / avoir chanté. Ce n'est pas un hasard si l'on a choisi des formes impersonnelles, car les descriptions de l'aspect sont trop souvent faussées par les interférences avec le temps. En tout cas, c'est certainement l'opposition aspectuelle le mieux représentée dans les langues du monde, et c'est pourquoi aussi les dénominations sont si variées. Ce qu'il faut absolument éviter, c'est d'appeler cette opposition imperfectif / perfectif. Or, en dehors des slavistes qui savent vraiment ce que recouvrent ces termes dans les langues slaves, les autres linguistes font, même involontairement, la confusion entre deux oppositions fondamentalement différentes ou tentent d'assimiler l'une à l'autre, ce qui, comme on le verra, est impossible. Il est préférable d'adopter une terminologie spécifique pour chaque type. Cela permet également de réserver le terme de parfait (angl. perfect) à la désignation d'un temps grammatical.

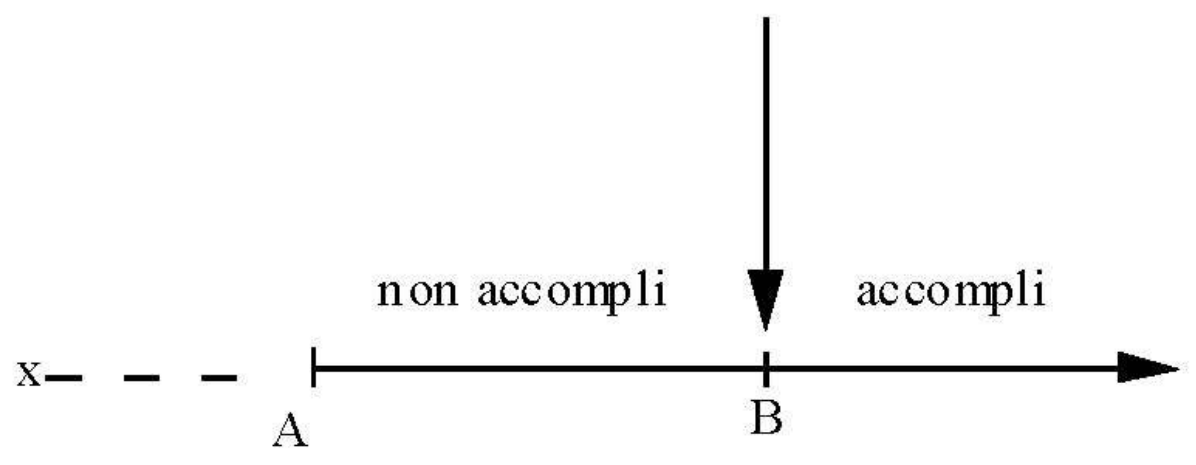

14 3) Un certain nombre de langues peuvent grammaticaliser l'opposition entre un procès saisi dans son développement et un procès qui ne spécifie pas ce trait. La représentation graphique sera la suivante (schéma nº3). 


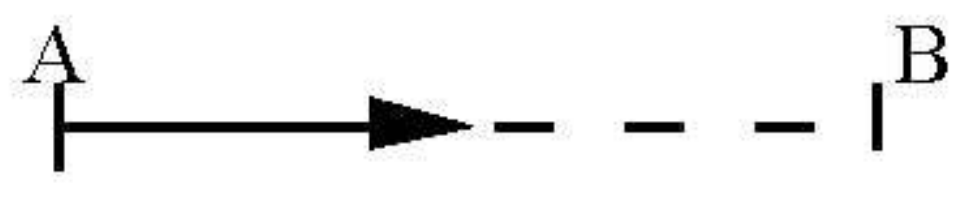
l'atteindra. Il faut éviter ici le terme de duratif opposé à celui de ponctuel, car s'il est vrai que le progressif suppose un déroulement qui dure quelque temps, le non-progressif n'est pas obligatoirement ponctuel. Au point de vue terminologique, l'opposition progressif / non-progressif peut être proposée comme terme générique, ce qui permettrait de faire l'économie de continu / non-continu (bien qu'en grammaire anglaise, on parle de continuous tenses) et surtout de continu / momentané qui présente les mêmes inconvénients que duratif / ponctuel.

Si une langue possède une opposition accompli / non-accompli, l'opposition de progressivité ne caractérise en fait qu'un des termes, à savoir le non-accompli : ce serait le cas en italien où la périphrase " être » + gérondif (ou participe dit « présent ») est limité au non accompli, car il y aurait une contradictio in terminis si l'on disait qu'une action accomplie est encore en train de se développer. Mais il y a un contre-exemple important : l'anglais. Dans cette langue, il existe une forme progressive à tous les temps de l'accompli. Or, il est clair qu'en ce cas, la forme progressive n'a pas sa valeur de base. Et c'est une des difficultés de l'aspectologie que de déterminer, à côté de la valeur de base, toutes les autres valeurs, dites souvent «secondaires», que peuvent prendre les formes aspectuelles : l'anglais en est la parfaite illustration.

Dans le cas du français, l'opposition entre l'imparfait et le passé simple est également de type sous-aspectuel dans la mesure où elle ne concerne que deux temps du système verbal et non l'ensemble. Il faut renoncer à la traiter en termes de duratif / ponctuel : rien n'indique dans Une heure sonnait quand il entra que la sonnerie dure plus longtemps que l'entrée. D'autre part, on n'a pas manqué de citer des exemples où le passé simple avait une valeur durative. Tesnière citait La guerre de Cent ans dura en réalité 116 ans, et Victor Hugo écrit sans difficultés Il marcha trente jours, il marcha trente nuits. C'est en termes de dynamisme qu'il faut poser le problème: dans un récit, l'imparfait a un caractère statique (il donne le cadre, l'arrière-plan, le décor), tandis que le passé simple a un caractère dynamique : c'est l'élément moteur qui fait progresser le récit en créant à chaque fois une nouvelle situation. Par conséquent, toutes les valeurs qu'on prête à l'imparfait, comme la durativité, l'itérativité ou l'habitude ne sont que des valeurs contextuelles qui se déduisent de la valeur de base et qui ne constituent pas des traits distinctifs.

4) Comme on l'a dit précédemment, l'opposition entre imperfectif et perfectif devrait être réservée à ce que l'on trouve avant tout dans les langues slaves. Bon nombre de linguistes n'ont pas manqué de faire remarquer, à juste titre, qu'elle était tout à fait particulière et que, contrairement à ce qui avait été dit, elle n'était nullement caractéristique de ce qu'on entendait en général par aspect. Sa représentation graphique n'est pas simple ; on proposera le schéma suivant (Feuillet 1996 : 88).

Linx, 45 | 2001 

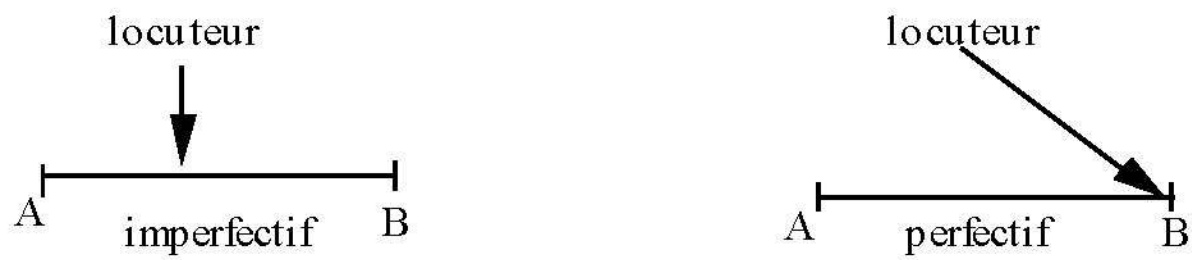

Veyrenc (1973 : 70) résumait ce type d'opposition aspectuelle d'une manière très concise : "L'aspect imperfectif présente le procès sans le spécifier. L'aspect perfectif implique au contraire une limitation du procès ». Cela signifie qu'avec l'imperfectif, le locuteur n'assigne pas de terme au procès, alors qu'avec le perfectif, il a en vue ce que $\mathrm{Zl}$. Guentchéva appelle la visée d'achèvement. On voit la différence avec l'opposition d'accomplissement : l'infinitif perfectif russe dat' n'a jamais le sens de « avoir donné » en face de l'imperfectif davat' qui signifierait « donner »; les deux verbes ont le sens général de "donner", et le français est en fait incapable de proposer deux traductions différentes.

Une autre preuve que l'opposition imperfectif / perfectif est différente de l'opposition non-accompli / accompli est que le système verbal bulgare possède les deux types : on trouve dans cette langue des imparfaits, des aoristes, des parfaits et plus-que-parfaits perfectifs et imperfectifs. Cette compatibilité mutuelle exclut une assimilation de tous ces types sous un seul terme générique.

21 Enfin, il se greffe un autre phénomène spécifique : l'opposition imperfectif / perfectif se fonde sur l'existence d'un couple de verbes, dont les rapports sont plus ou moins grammaticalisés. C'est un critère fondamental pour poser une opposition grammaticale. En effet, si les couples verbaux ne reposaient que sur une opposition entre verbe simple et verbe préverbé, cela ressortirait au lexique. Mais le fait que les langues slaves puissent reformer, grâce à un suffixe, un imperfectif dérivé possédant exactement le même sens que le perfectif préverbé et formant avec ce dernier un couple dit de « corrélation », est le signe que la grammaticalisation est complète. En vérité, aucune langue indo-européenne autre que slave ne connaît un tel système. On pourrait parler cependant d'une opposition imperfectif / perfectif si une langue donnée se servait toujours du même préverbe délexicalisé pour donner naissance à un couple verbal. C'est la situation de l'allemand médiéval où, à un verbe simple, s'oppose un verbe à préfixe ge-. Effectivement, les valeurs qu'on s'accorde à trouver à cette opposition concordent bien avec celles que l'on trouve en slave. Le meilleur terme pour désigner cette opposition est celui de limitation.

On peut imaginer un autre type de découpage, celui qui consiste à parler de phases du procès. Dans les langues indo-européennes et dans bien d'autres familles, les phases sont indiquées par des moyens lexicaux ("commencer », «continuer », " finir ») ou par des préverbes. On parle en ce cas d'ingressif ou d'inchoatif pour l'entrée dans le procès, de continuatif pour la poursuite du procès et de terminatif ou d'égressif pour la sortie du procès, l'achèvement impliquant en même temps la sortie. La représentation graphique aurait cette forme : 


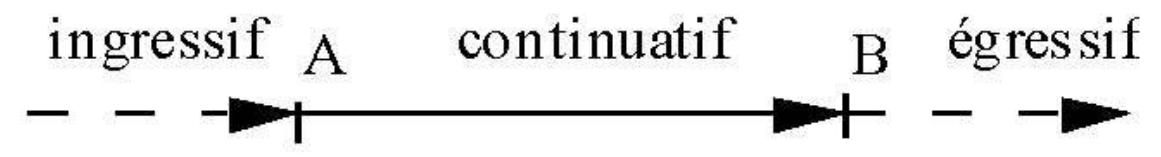
indique un morphème -wa dans patsena-wa qu'il traduit par « he is going on doing ». Mais les autres formes verbales révèlent un système mixte : un progressif xoo-i « he is crying ", un habituel muf-maa « he smokes (as a habit) », un incomplétif kwaj-m? $f a$ « he will arrive » et un ponctuel tfup $\Phi k^{\prime}$ oj-pa « he entered in ». Pour les langues bantu, Alexandre (1981: 361-362) indique les valeurs aspectuelles d'inceptif, d'itératif, de fréquentatif, de continu, mais ne donne pas d'exemples qui montreraient comment les systèmes aspectuels s'organisent. C'est très souvent le cas en typologie lorsqu'on a recours à des descriptions de langues variées : on donne une liste de suffixes avec différentes valeurs aspectuelles, aspectives, temporelles, modales, mais on n'indique pas en général les compatibilités entre les divers affixes, de sorte qu'on ne sait pas ce qui relève de la grammaire et ce qui relève du lexique. En tout cas, jusqu'à preuve du contraire, il semble qu'aucune langue ne grammaticalise dans son système aspectuel les différentes phases d'un procès, de sorte que les schémas qu'on a proposés peuvent s'appliquer pour toutes les langues qui connaissent des oppositions aspectuelles.

Ce sont donc les différentes manières dont les langues découpent ou envisagent le procès qui constituent les véritables oppositions aspectuelles. On parlera ici d'aspect interne qu'il convient de distinguer des caractères externes que l'on va aborder maintenant.

\section{Aspect « externe » et valeurs complémentaires}

Parmi tous les termes qui sont utilisés dans les descriptions de l'aspect, certains renvoient à des traits qui ne concernent pas la division interne du procès. C'est ainsi qu'on parle d'itératif, d'habitatif, de répétitif, de fréquentatif ou d'intensif. Doit-on considérer qu'il s'agit de caractères relevant de l'aspect? La réponse, sous bénéfice d'inventaire, serait plutôt négative, mais il n'est pas exclu que certaines langues aient grammaticalisé des suffixes. Malheureusement, comme on l'a fait remarquer précédemment, les descripteurs n'indiquent généralement pas les compatibilités : on en est donc souvent réduit à des interprétations qui peuvent se révéler fausses. La question fondamentale est de savoir si l'affixation permet la création d'un nouveau verbe qui posséderait, comme le verbe simple, toutes les formes verbales (aspect, temps, mode) ou si, au contraire, l'affixe fait partie du verbe lui-même en tant qu'élément grammaticalisé. Si l'on prend par exemple l'arabe, il est clair que les neuf formes (vivantes) dérivées d'une racine ne ressortissent pas à l'aspect, mais au lexique, puisque chaque verbe dérivé est soumis à l'opposition aspectuelle inaccompli / accompli. Par conséquent, si l'on considère kasara « briser » à côté de la forme dérivée II kassara « briser en petits morceaux » ou de la forme dérivée V takassara «se briser» (Lecomte 1968: 28-30), on ne peut parler d'opposition aspectuelle entre le verbe simple et les verbes dérivés. Toute caractérisation $\mathrm{du}$ procès qui ne repose pas sur une saisie interne n'appartient donc pas à l'aspect proprement dit: en exprimant la répétition ou l'intensité d'un procès, on ajoute des 
éléments extérieurs. Que le procès soit une habitude (et donc qu'il soit susceptible de se répéter) ou qu'il se produise fréquemment ne le caractérise pas dans son déroulement ou dans son achèvement. Ces valeurs ne peuvent pas être mises sur le même plan que les autres, et on doit donc les rattacher à l'aspectivité. Elles équivalent aux valeurs véhiculées par des adverbes temporels comme « fréquemment, souvent, habituellement » ou par des verbes distincts ("avoir l'habitude de», allem. pflegen, angl. au prétérit he used to) ou encore par des préfixes, comme allem. wieder-, français re-.Le tchèque se sert d'un suffixe -va- comme fréquentatif, mais il s'agit en réalité d'un autre verbe que le verbe simple. L'irlandais oppose bím « je suis habituellement » à táim « je suis » (cf. bulg. bivam et săm, russe à l'infinitif byvat' et byt'). Ce phénomène est donc de nature lexicale. Il n'empêche que certaines formes verbales peuvent exprimer l'habituel : c'est le cas en anglais de would + infinitif ou du conditionnel en haut-sorabe. La solution est de faire de ce fréquentatif une valeur secondaire ou annexe de la forme verbale, tout comme on accorde une valeur de modalisation au futur antérieur, comme dans Il n'est pas encore arrivé, il se sera égaré. On notera qu'en anglais ou en sorabe, cette valeur est attachée à une forme verbale finie.

Cette remarque n'est pas une manière élégante d'évacuer les faits dérangeants. En effet, on peut constater qu'à côté des valeurs aspectuelles que l'on peut déterminer objectivement en envisageant toutes les possibilités de découper le procès (segment $\mathrm{AB}$ ), il existe un grand nombre de valeurs dites «modales » qui sont indépendantes. La forme en -ing de l'anglais est révélatrice à cet égard. On peut rendre compte de la valeur de cursif (ou de progressif) en termes d'aspect: He is smoking «Il est en train de fumer». Mais il est impossible d'utiliser la même explication dès qu'il s'agit d'analyser les autres valeurs de la forme en -ing, qui sont souvent délicates à saisir. Le plus frappant peut-être est que la périphrase n'est jamais une servitude grammaticale. On peut résumer ainsi les emplois les plus courants (König 1994 : 544) :

- Cursif pour l'action qui se déroule sans avoir atteint son terme. C'est la valeur de base : He is working / smoking «Il est en train de travailler / de fumer ».

- Réitération: The baby has been falling out of its bed "Le bébé est tombé de son lit" (plusieurs fois) / The baby has fallen out of bed « Le bébé est tombé du lit » (action unique).

- Effets concomitants (alors que la forme non progressive indiquera plutôt le résultat) : I have been mowing the lawn / I have mowed the lawn «J'ai tondu le gazon ».

- Quantité indéfinie: It has been snowing a lot "Il a pas mal neigé " / It has snowed two inches «Il a neigé deux pouces » (quantité définie).

- Incomplétude : Perhaps we have been spoiling her a little « Peut-être l'avons-nous gâté un petit peu» / We have spoilt her a little (complétude).

- Spécification de la durée de l'activité plutôt que l'état résultant: They have been leaving for half an hour / They have left for half an hour « Ils sont partis il y a une demi-heure ».

Il faut évidemment mettre à part les périphrases où for a le sens de « depuis », car il s'agit de vrais présents : I have been living in Paris for ten years «J'habite à Paris depuis dix ans ».

En bulgare, dans la concurrence aspectuelle entre l'imperfectif et le perfectif, l'imperfectif peut apporter des nuances complémentaires, comme celle d'attitude négative vis-àvis du procès ou d'invitation à entreprendre l'action. Voici des exemples des deux valeurs :

(1) S žena-ta rešixme Šte Se razveždame. To šte 


\begin{tabular}{|l|lll|}
\hline & avec femme-la décidâmes FUT RÉFL divorçons (IMPERF) cela FUT \\
\hline \hline & $e$ razvod na užkim, ama inače ne može (X. Xristov) \\
\hline & est divorce en apparence mais autrement NÉG peut \\
\hline
\end{tabular}

« Avec ma femme, c'est décidé... Nous allons divorcer. Ce sera un faux divorce, mais on ne peut pas faire autrement ».

[Le contexte montre clairement qu'ils n'ont pas envie de divorcer].

\begin{tabular}{|l|lllllllll|}
\hline$(2)$ & Riskuvam & $d a$ & zapaljat & čerga-ta & mi! & Plaštaj & ili šte obrǎštam & & \\
\hline & je risque & que incendient & carpette-la & POSS & paie & ou & FUT & rends \\
\hline & kone-te & (N. Kirilov) & chevaux-les & & & & & & \\
\hline
\end{tabular}

«Ma maison risque d'être incendiée! Paie ou je vais rendre les chevaux ».

Il est clair que l'aspectologie ne peut faire l'impasse sur les valeurs secondaires véhiculées par les formes qui entrent en concurrence, mais il est non moins clair qu'elle doit toujours accorder la priorité à la détermination des valeurs de base.

Cet exposé n'a pas la prétention d'apporter des réponses définitives aux nombreuses questions que soulève le traitement des oppositions aspectuelles: il se veut surtout un essai de bien distinguer les niveaux d'analyse, c'est-à-dire de ne pas mélanger tous les faits, mais de les classer. Il faut avant toute chose comparer un grand nombre de systèmes avant de se lancer sans précaution dans des généralisations sémantiques. Savoir comment fonctionnent les systèmes aspectuels, séparer les valeurs de base des valeurs secondaires, créer une terminologie adéquate en évitant les assimilations de tel système à tel autre, voilà les exigences qui sont ou devraient être celles de l'aspectologue.

\section{BIBLIOGRAPHIE}

ALEXANDRE Pierre 1981 : « Les langues bantu », in PERROT Jean (éd.), p. 351-397.

COHEN David 1989 : L'aspect verbal, Paris, P.U.F.

COMRIE Bernard 1976 : Aspect, Cambridge, Cambridge University Press.

FEUILLET Jack 1983 : “Methodologische Probleme des Aspekts", Ziele und Wege der Balkanlinguistik (herausgegeben von Norbert Reiter), Wiesbaden, Harrassowitz, p. 66-80.

FEUILLET Jack 1996 : Grammaire synchronique du bulgare, Paris, Institut d'Études Slaves.

HUMBERT Jean 1972 : Syntaxe grecque, Paris, Klincksieck. 
KÖNIG Ekkehard « English » in KÖNIG Ekkehard \& VAN DER AUWERA Johan (eds) 1994 : The Germanic Languages, London-New York, Routledge, p. 532-565.

LECOMTE Gérard 1968 : Grammaire de l'arabe, Paris, P.U.F., coll. « Que sais-je? ».

PERROT Jean (éd.) 1981 : Les langues dans le monde ancien et moderne. Afrique subsaharienne. Pidgins et créoles, Paris, Éditions du CNRS.

POTTIER Bernard (sous la direction de) 1973 : Le langage, Paris, Denoël.

SUÁREZ Jorge 1983 : The Mesoamerican Indian languages, Cambridge, Cambridge University Press.

ULRICH W. 1972 : Linguistische Grundbegriife, Kiel, Verlag Ferdinand Hirt.

VEYRENC Charles Jacques 1973: Grammaire du russe, $2^{\mathrm{e}}$ édit., Paris, P.U.F.

\section{AUTEUR}

JACK FEUILLET

INALCO-PARIS 